\title{
Effect of Predatory Stress Before Fertilization on Body Weight and Pentylenetetrazole-Induced Seizure in Rat Offspring
}

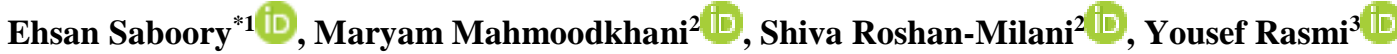

1. Zanjan Metabolic Diseases Research Center, Zanjan University of Medical Sciences, Zanjan, Iran

2. Neurophysiology Research Center, Urmia University of Medical Sciences, Urmia, Iran

3. Cellular and Molecular Research Center, Urmia University of Medical Sciences, Urmia, Iran

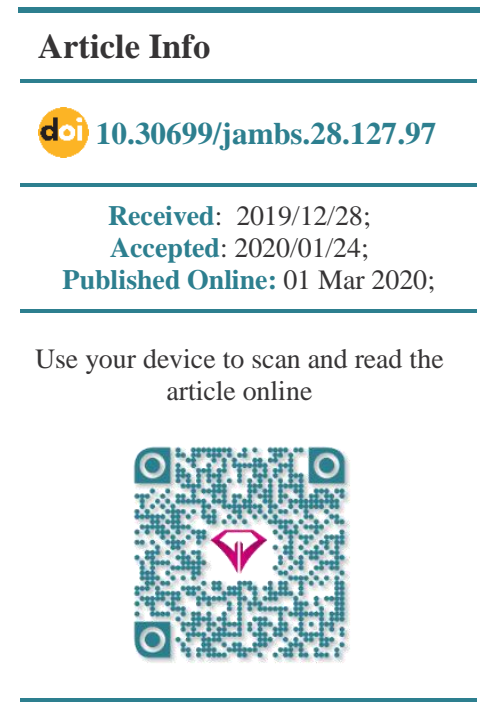

Corresponding Information:

Ehsan Saboory,

Metabolic Diseases Research Center, Zanjan University of Medical Sciences, Zanjan, Iran

E-Mail: saboory@zums.ac.ir

\section{ABSTRACT}

Background \& Objective: Spermatogenesis and oogenesis stress is a primary epigenetic element that can cause lifelong modifications in neural system functions. This study was designed to elucidate whether preconceptional stress during spermatogenesis and oogenesis affects the seizure behaviors and body weight of the offspring later in life.

Materials \& Methods: In this experimental study, 16 male and 32 female adult rats were included. Half of the rats were subjected to predatory stress. Then, they were coupled as follows: both subjects were intact as the control group (MC-FC), both subjects were stressed (MS-FS), male control/female stressed (MC-FS), and male stressed/female control (MS-FC). The pups were weighed and assessed for pentylenetetrazole-induced seizures. In addition, aniline blue staining was performed to study the chromatin maturity of the sperm in male parents immediately after copulation.

Results: The latency of the first seizure behavior significantly diminished in stressed pups, and the duration of focal and generalized seizures significantly increased in the stressed rats .In addition, the body weight of the pups decreased in the stress groups compared to control rats, and a significant decrease was detected in the chromatin maturity of sperms in stressed rats.

Conclusion: Stress during spermatogenesis and oogenesis can be critical for the general growth (body weight) and seizure susceptibility of the offspring. Therefore, to improve reproductive outcomes, stress-lowering interventions are better to be started before conception.

Keywords: Body weight, Preconceptional, Stress disorder, Rats, Seizures

\section{Introduction}

Organisms naturally face perturbations threatening their immediate survival possibly affecting their main life-history features (1). The quality of the social environment may have considerable effects on the structure and function of nervous systems with implications for several behavioral and physiological responses $(2,3)$. One of the most important environmental factors is stress which is unavoidable and experienced by all and can be psychological, physical, or both (4). Stressors happening through the critical stages of development, for instance, the perinatal period, may disturb physiological functions such as metabolism, reproduction system, and growth (5). In humans, the influence of early-life stress on the brain structure and function has been revealed to last a long time after stress exposure, possibly up to adulthood. Exposure to earlylife stress raises the risk of neuropsychiatric illnesses, including epilepsy (6). Stress not only increases seizure susceptibility at times of stress but also the risk of the development of epilepsy, especially in persons who have experienced early-life stress. It has been reported that prenatal stresses (PS) potentiate seizure behaviors and raise the vulnerability to seizures in rat offspring (7). In addition to the effects of PS on the first generation, several studies have mentioned the transfer of stress to the second generation (6). One likely explanation for the persistence of dynamic changes in these systems in the response to environmental factors is the participation of epigenetic mechanisms (2). Epigenetics has been used more broadly to refer to any change in gene function not associated with sequence variation. Epigenetic processes essentially influence brain development. Insults in maturation throughout pregnancy might be expected to more broadly re-program the epigenome; if they are incorporated into the germ cells, their effects will possibly become trans-generational (2). Furthermore, epigenetic characters generated within germ cells due to environmental influences all over life can shape future generations long before conception occurs (2). The connection between epigenetics and human 
reproduction characterizes a very interesting field of study, generally due to the possible trans-generational effects related to epigenetic modifications of male and female gametes (8). The integrity of the sperm genome is vital for the accomplishment of a normal conception in addition to the typical growth of the fetus and children (9). In addition, it is proven that the quality of the oocyte determines the potential development of the embryo (6).

The present study was designed to elucidate whether pre-gestational stress during spermatogenesis and oogenesis (particularly chromatin changes in sperms) affects the seizure behaviors and body weight of the offspring later in life.

\section{Materials and Methods}

\section{Animals}

Wistar rats of both sexes (190-240 g) were purchased from the Urmia University of Medical Sciences, West Azerbaijan, Iran. The subjects were housed at a $12 \mathrm{~h}$ light/dark cycle (light on at 7 a.m.) and the temperature of $20-24^{\circ} \mathrm{C}$, and food and water were available ad libitum (6). After seven days of adaptation, the rats (16 adult males and 32 adult females) were randomly assigned into two groups (control and stressed male, $\mathrm{n}$ $=8$ each; control and stressed female, $\mathrm{n}=16$ each) to form a combination of control and stressed groups for each sex.

\section{Predatory Stress Procedure}

A healthy adult cat was used for inducing predatory stress in the rats. As described in our previous study, the procedure was as follows: A metal cage $(72 \times 72 \times 63$ $\mathrm{cm})$, with a cat inside, was placed in front of the rats' cages (Figure 1). The cage of the cat consisted of a metal floor with a removable hinged door and several small air holes on the side, so that the cat could be easily observed. To induce predatory stress, the rats were positioned in metal mesh cages $(20 \times 22 \times 22 \mathrm{~cm})$ with numerous holes in the lateral walls. The cages banned any direct contact between the predator (cat) and the rats but exposed the rats to other stimuli, e.g. the vision, smell, and noises made by the cat (6). The subjects were stressed at 8-9 a.m. and 4-5 p.m. (twice daily) for 50 or 15 consecutive days (males and females, respectively). Immediately after each session, the rats were moved to their home cage. The rats in the control group were transported to another room without any cat odors and handled similarly to the stressed rats but without being stressed. The stress procedure lasted for 50 days (in males) because it is the time required for a complete spermatogenesis cycle in male rats, while 15 days constituted three cycles of the estrous cycle in female rats $(\mathbf{1 0})$.

Immediately after the stress procedure, the rats were assigned for copulation. The copulation method was similar to that explained in our previous study: Two female rats were coupled with one male rat for three days (the Trichus method) per cage as follows: Mc-Fc (both rats were control), Ms-Fc (the male was exposed to stress, but the female was control), Mc-Fs (the male rat was control but the female was stressed), and MsFs (the two rats were stressed) (10).

The pregnant subjects were transferred to new cages and kept in normal conditions for the entire pregnancy period. The duration of pregnancy was the same, 21 days, in four groups. Upon delivery, the pups were counted, weighed, and gendered. All the litter was culled to eight per dam. The rat pups were allocated according to their own parents' grouping as follows: Both fathers and mothers were control $(\mathrm{Mc}-\mathrm{Fc})$; just the mother was stressed (Mc-Fs); just the father was stressed (Ms-Fc); and both the father and mother were stressed (Ms-Fs) (10). There were 64 pups in each group $(8$ dams $\times 8$ pups $=64)$.

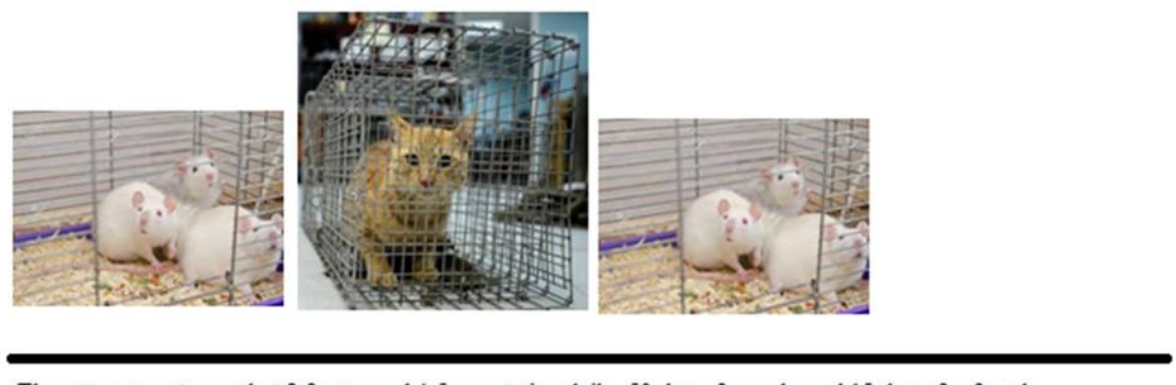

The rats were stressed at $8-9$ a.m. and $4-5$ p.m, twice daily, 50 days for male and 15 days for female.

Figure 1. Schematic illustration of the predatory stress procedure.

\section{Sperm Sampling in Adult Rats}

Under anesthesia with isoflurane, the stressed and control male parents were subjected to sperm sampling in the morning ( $\mathrm{n}=8$ per group) after copulation. Both epididymides of each rat (at the caudal region) were transferred to a glass Petri dish containing an $80 \%$ DMEM/F12 (DMEM/F12; ATCDHF, St. Louis, USA) culture supplemented with a $20 \%$ fetal bovine serum
(FBS; Sigma, St. Louis, USA) medium pre-warmed to $37 \mathrm{oC}$. The epididymides were crushed, and six cuts were made with an insulin syringe. The samples were incubated for $30 \mathrm{~min}$ at $37^{\circ} \mathrm{C}$ in $5 \% \quad \mathrm{CO}_{2}$; then, spermatozoa were released from the epididymides (10). 


\section{Chromatin Quality and Acidic Aniline Blue (AB) Staining}

For each neat semen sample, the sperm nuclei chromatin condensation was evaluated using $\mathrm{AB}$ staining. Aniline Blue selectively dyes histones with abundant lysine and can demonstrate the sperm chromatin irregularities associated with histone anomalies. Fixations of air-dried smears of rat semen samples were prepared in buffered glutaraldehyde (3\%) in a phosphate buffer $(0.2 \mathrm{M}$ and $\mathrm{pH}$ 7.2) for half an hour at $22-24^{\circ} \mathrm{C}$. Then, the smears were stained with aqueous $\mathrm{AB}$ stain (5\%) in acetic acid (4\% and $\mathrm{pH}$ = 3.5) for $7 \mathrm{~min}$ (11). In a stained smear, 200 sperm were studied under a light microscope with $\times 100$ magnification. In this staining, the spermatozoa with light-blue nuclei are considered as normal, and those with dark-blue nuclei are counted as abnormal (12).

\section{Body Weight Measurement}

Upon delivery, the rat pups for each dam were counted and weighed in the morning at 08:30 on the first postnatal day (P1). Weighing of the pups was repeated at $08: 30$ on postnatal days $6,15,25$, and 30 (P6, P15, P25, and P30, respectively).

\section{Behavioral Assessment}

In the pentylenetetrazole (PTZ) model, seizures are commonly induced by a single systemic administration, and PTZ can even induce status epilepticus if given in a sufficient amount (13). On P30, one male and one female pup from each dam were subjected to seizure induced by PTZ. Then, the pups were intraperitoneally (IP) injected with PTZ $(55 \mathrm{mg} / \mathrm{kg})(6,14)$. After injection, the behavior of each rat was assessed for one hour with a digital camera and direct observation. The behavioral rating was evaluated by means of a previously defined scale: normal behaviors $=0$; immobilization with or without sniffing $=1$; forelimb clonus with head nodding named as short myoclonic jerk $=2$; tail extension and nonstop myoclonic jerk $=3$; widespread limbic seizures with kangaroo position $=4$; and nonstop tonic or tonic-clonic seizures $(15,16)$. Other variables were also evaluated as follows:

Time to the onset of the first epileptic behavior (sec): time interval between the injection of PTZ and the appearance of the first seizure; the number of focal seizures: the number of focal seizures in 60 minutes (e.g. leg opening, immobility, facial twitching, ...); duration of focal seizure (min), total time of all focal seizures in 60 minutes; the number of tonic-clonic seizures, the number of tonic-clonic seizures in 60 minutes that each rat experienced; duration of tonic-clonic seizure (sec), total time of all tonic-clonic seizures in 60 minutes

\section{Ethical Consideration}

This experimental study was approved by the Ethics Committee in West Azerbaijan Province, Iran (ethical code: IR.1395.229).

\section{Statistical Analysis}

Data distribution was checked by the KolmogorovSmirnov test. The normally distributed data were analyzed using one-way ANOVA (body weight and epileptic behaviors, except for the number of focal seizures and the onset of the first seizure) followed by Tukey's post-hoc test when required. The data related to the latency (onset) of the first seizures and the number of focal seizures were evaluated by KruskalWallis or Mann-Whitney U test. Also, t-test was performed for the comparison of chromatin maturity between stressed and control rats. The findings were stated as mean \pm standard error of the mean (SEM), and the P-value $<0.05$ was considered significant.

\section{Results}

\section{Effects of Pregestational Stress on Seizure}

On P30, PTZ-induced seizure was assessed in rat pups. First, the data of the pups for male and female pups were independently analyzed; no significant difference was detected between the two sexes. Then, the data related to the seizure of male and female pups were combined and evaluated together (Table 1).

\section{Effects of Pre-conception Stress on Bodyweight in Rat Pups}

There were significant differences in body weight among the groups at different time points (Table 2). In pregestational stress, the mean body weight of the offspring significantly decreased at birth $(\mathrm{P} 1, P=0.03)$ and P6 $(P=0.02)$ compared to the control pups.

\section{Effects of Stress During Spermatogenesis on Chromatin Maturity}

Remarkable differences were found in the chromatin maturity of sperms between stressed and control adult rats (Figure 2). Also, an illustration of sperms with normal and abnormal chromatin maturity is shown in Figure 3.

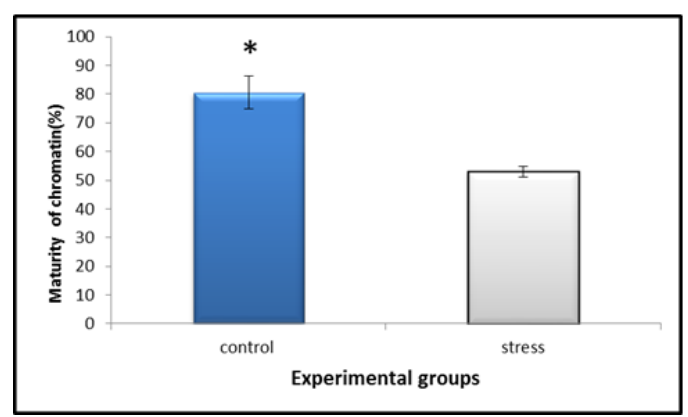

Figure 2. Effect of stress during spermatogenesis on the chromatin maturity of sperms in adult rats; *indicates $\mathbf{P}=0.004$ with the stress group. 


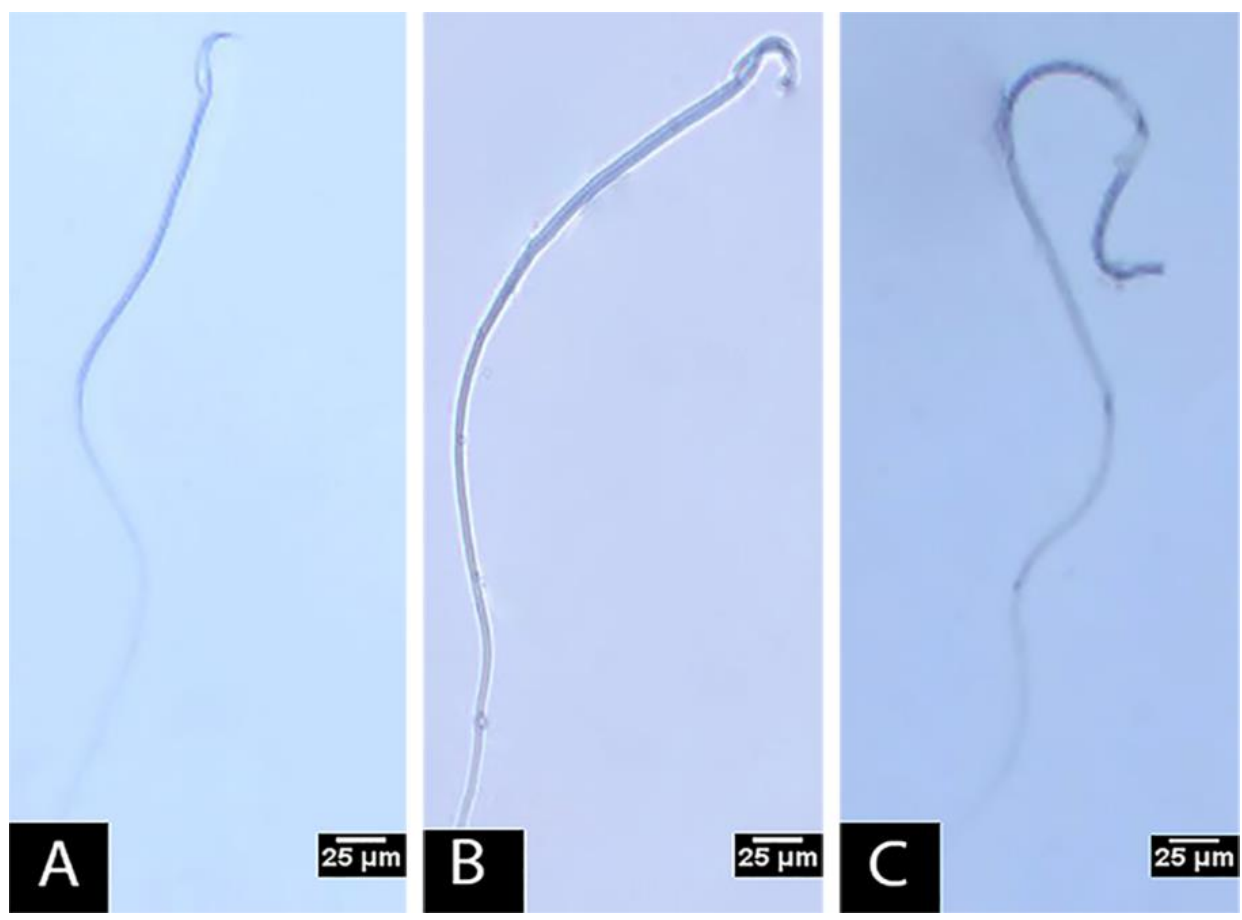

Figure 3. Aniline blue staining of sperms in adult Wistar rat subjected to predatory stress for 50 consecutive days; (A) a normal sperm; (B and C) sperms with abnormal maturity $(\times 1000)$.

Table 1. Effect of pre-conception stress during spermatogenesis and oogenesis on PTZ-induced seizure in the rat pups

\begin{tabular}{llcccc} 
Epileptic behaviors & \multicolumn{5}{c}{ Pups grouping } \\
\cline { 2 - 6 } & MC-FC & MC-FS & MS-FC & MS-FS & P-value \\
$\begin{array}{l}\text { Time to onset of first } \\
\text { epileptic behavior (Sec) }\end{array}$ & $119.87 \pm 14.61$ & $\# 18.25 \pm 1.95$ & $\# 56.50 \pm 6.48$ & $\# 11.37 \pm 0.53$ & $\# P<0.01$ vs. Mc-Fc \\
\hline $\begin{array}{l}\text { Number of focal seizure } \\
\text { Duration of focal seizure }\end{array}$ & $1.5 \pm 0.32$ & $2.12 \pm 0.35$ & $1.75 \pm 0.36$ & $2.12 \pm 0.35$ & non-significant \\
(min) & $9.25 \pm 2.54$ & $14.62 \pm 2.01$ & $* 20.12 \pm 2.17$ & $* 16.251 \pm 1.17$ & $* P<0.04 \mathrm{vs.} \mathrm{Mc-Fc}$ \\
$\begin{array}{l}\text { Number of tonic-clonic } \\
\text { seizure }\end{array}$ & $0.5 \pm 0.18$ & $1.12 \pm 0.26$ & $1.12 \pm 0.22$ & $2.75 \pm 0.61$ & non-significant \\
\hline $\begin{array}{l}\text { Duration of tonic-clonic } \\
\text { seizure (Sec) }\end{array}$ & $1.37 \pm .53$ & $* 13.75 \pm 3.09$ & $* 14.87 \pm 2.61$ & $* 22.62 \pm 4.57$ & $* P<0.03$ vs. Mc-Fc \\
\hline
\end{tabular}

Data presented as mean \pm SE: \# non-parametric Kruskal Wallis test, * Tukey post-hoc test.

MC-FC: both parents' control, MC-FS: male control/female stressed, MS-FC: male stressed/female control, MS-FS: both parents stressed

Table 2. Effects of pre-gestational stress on bodyweight in rat pups

\begin{tabular}{cccccc} 
Groups & \multicolumn{3}{c}{ Body weight } \\
& P1 & P6 & P15 & P25 & \\
Mc-Fc & $6.45 \pm 0.65$ & $11.59 \pm 0.22$ & $23.61 \pm 0.69$ & $42.49 \pm 1.1$ & $56.27 \pm 0.95$ \\
Mc-Fs & $* 6.15 \pm .09$ & $* 10.36 \pm .37$ & $21.15 \pm 0.58$ & $39.93 \pm 1.43$ & $41.43 \pm 0.47$ \\
Ms-Fc & $6.38 \pm 0.09$ & $11.24 \pm 0.12$ & $21.43 \pm 0.26$ & $39.84 \pm 0.66$ \\
Ms-Fs & $\# 5.45 \pm 0.11$ & $* 10.32 \pm 0.19$ & $20.99 \pm 0.58$ & $53.36 \pm 0.7$ \\
P-value & $* P<0.03$ vs. Mc-Fc & $P<0.02$ vs. Mc-Fc & non-significant & non-significant & non-significant
\end{tabular}

Data presented as mean \pm SE and analyzed by One-way ANOVA and Tukey tests.

P1. postnatal day1, P6. postnatal day6, P15. postnatal day15, P25. postnatalday25, P30. postnatal day30

MC-FC: both parents control, MC-FS: male control/female stressed, MS-FC: male stressed/female control, MS-FS: both parents stressed

\section{Discussion}

The effects of pre-gestational stress on PTZ-induced seizure, the body weight of the offspring, and the sperm chromatin changes of male parents were investigated.

Volume 28, March \& April 2020
The main findings were that pregestational stress caused a significant increase in generalized seizures and decreased the body weight of the offspring at birth

Journal of Advances in Medical and Biomedical Research 
(P1) and on P6. In addition, there was a significant decrease in the percent of spermatozoa with mature chromatin in the stressed rats compared to the control group.

\section{Effects of Pre-conception Stress on Epileptic Behaviors in Rats}

The results demonstrated the negative effects of prepregnancy stress on the offspring. In this regard considerable evidence exists supporting the fact that many factors can persuade hostile effects in the descendants following parental exposure and, consequently, represent a threat in this respect (17). PS can be regarded as a primary epigenetic element able to cause lifelong modifications in neural system functions (18). In previous studies, the effect of early stress on growth has been studied; early-life stress, such as prenatal stress, is reported to be proconvulsant. In one study, PS amplified seizure and diminished the latency of seizure from early infancy up to later life in a kindling epilepsy model (19). Moreover, a vital element influencing adult nervous systems (such as the hippocampus, amygdala, and the hypothalamicpituitary-adrenal (HPA) axis) is the exposure of parents to noticeable stimuli in the environment before pregnancy (20). Notably, the trans-generational influence of the chronic stress of parents is not limited to the perinatal window; alterations in the descendants' stress-associated behavior have been stated following the exposure of parents to stress up to adulthood (21). The findings of our study are consistent with the results of previous investigations. The mechanisms by which long-lasting parental stress can disturb the descendants' neurodevelopment are not fully understood yet. A mechanism explaining the impact of pre-pregnancy stress on the offspring is the HPA axis. Environmental factors, mainly a positive stress history, contribute to the developmental programming of the HPA axis (21). Increasingly, the HPA axis is being considered as a compound physiological system able to facilitate huge variations in the physiological responses of subjects to stressors (1). It has been reported that a repetitive experience of PS-induced disruption in the function of the HPA axis and modification of its feedback regulation originate a greater basal secretion of glucocorticoid hormones (22). Furthermore, it has been indicated that the serum corticosterone and adrenocorticotropic hormone $(\mathrm{CRH})$ levels were higher in maternal rats with pre-gestational chronic unpredictable stress (CUS) than in the control group, both after CUS and delivery. Chronic stress not only leads to the imbalance of the neuroendocrine network of maternal rats but also impacts the HPA axis of the fetus via the maternal-placental-fetal interface (23).

Previous studies have argued that stress before gestation will possibly affect the hippocampal maturation and impair the memory function of the offspring, and cause reduced brain-derived neurotrophic factor levels (24). Perhaps the same argument applies to the effect of pre-pregnancy stress on the offspring's epileptic behaviors. Meanwhile, it has been shown that stress disturbs the integrity of the sperm chromatin (4) which is important as it indicates that sperms possibly pass damaged genes onto the zygote and embryo which may eventually influence the health of the descendants (25). In the present study, in groups where the father was stressed, the chromatin maturation rate decreased and the health of the children was affected. Also, research on some animal species indicates that egg quality is effective on the growth and health of the fetus (26). Here, the stress state in the prepregnancy period in parents showed that stress during gametogenesis can transfer to the offspring, thereby altering the offspring's vulnerability to seizure. In conclusion, the present study demonstrated that the exposure of rats to stress during spermatogenesis and oogenesis can change their next generation's vulnerability to PTZ-induced epileptic behaviors later in life.

\section{Effects of Pre-conception Stress on the Body Weight of the Offspring}

Studies have reported that PS leads to a low birth weight. Animal studies have demonstrated that exposing animals (such as rats and mice) to stressors during pregnancy is associated with lower birth or fetal weights. Maternal stress affects the growth and organ development of fetuses, targeting some organs more than others, strikingly attenuating glucose transporter expression, and diminishing fetal plasma glucose, growth hormone, and $\mathrm{CRH}$ levels (27). There have been a few experiments examining the effect of stress before pregnancy on the pups' body weight. According to the findings, a concomitant decrease in the weight of dams and their fetuses indicates that growth retardation will possibly relate to the dams with CUS before pregnancy (24). As our study showed, in the case of mothers exposed to stress during oogenesis, their offspring showed a lower body weight on P1 and P6. Ronnenberg et al. studied the connection between pre-gestation body mass index and delivery outcomes among women in China; children born to women who were underweight before gestation were at a higher risk for fetal growth retardation (28). Another study assessed the welfares of prescribing iodized oil to women before pregnancy or during early pregnancy in Algeria. The children of treated mothers had a significantly greater birth weight compared to control women (29). These studies, in line with our study, show that maternal conditions before pregnancy can affect the health and weight of the offspring. On the other hand, the findings of another study identified no difference in the body weight of the offspring of subjects undergoing pre-gestational stress and the controls (24), a finding that contrasts those of the present study. Also, it has been reported that sexual behavior can be the most susceptible feature of male reproduction to stress due to the antagonistic association between male sex hormone and glucocorticoids. This principle of sperm quality might predict the treatment success as recommended by its links at several issues in the reproductive course, such as interrupted 
preimplantation of the embryo and birth defects in the children (30). The mechanisms by which pregestational stress affects pup growth remain largely unknown. Nevertheless, pregestational CUS leads to a remarkable rise in GC such as corticosterone levels in the offspring of the stressed subjects (24). Also, an animal study suggests that periconceptional undernutrition may influence the HPA axis which, in turn, influences the outcomes (28). However, the long-term influence of stress has been connected to a disruption in the HPA axis physiology and a change in its negative feedback regulation, affecting fetal growth and development (22). Our findings indicated that in the case of mothers exposed to stress during oogenesis, their offspring showed a lower body weight at birth and six days after birth. This result is consistent with the findings of the above-mentioned studies, suggesting that parental stresses before conception affect the birth weight and early neonatal body weight of the newborns (10). Meanwhile, to the best of our knowledge, this study is unique because both parents' stress and the single-parent (maternal or paternal) stress before conception were investigated on seizure susceptibility and body weight in the offspring, whereas previous studies have been conducted on single parents (mother or father alone).

\section{Effects of Pre-conception Stress on Sperm Chromatin Integrity}

The successful fertilization of eggs and subsequent development of the offspring will greatly depend on the quality of gametes produced by the parents. A previous study has indicated that stress has a negative impact on various parameters (such as sperm morphology and motility) associated with semen quality (10). A growing body of evidence connects the DNA damage of the sperm with mutation-related hazards and disorders in the offspring (31). Additionally, researchers believe that elevated psychological stress can be linked with amplified oxidant agents' synthesis, and chronic stress exposure can raise the production of reactive oxygen species (ROS) (32). It has been shown that oxidative stress affects sperm chromatin integrity and leads to single- and/or double-strand DNA breaks (33). Meanwhile, sperms are not able to reinstate the oxidative stress-induced impairment since they lack the essential enzymatic repair systems. This weakness is a criterion which makes the spermatozoa very vulnerable to oxidative damage (33). Defects in the male genome have been shown to lead to post-fertilization failure (34). Previous studies recommend that DNAfragmented sperm can cause several reproductionrelated problems such as conception failure, abortions, and malformations (32). Thus, the findings of the present study confirmed the result of previous studies showing that pregestational stresses disturb the maturity of the chromatin of sperms in stressed subjects $(32,33)$. In conclusion, a precise assessment of the stress status and seminal ROS levels should be considered as an integral part of the reproduction workup of men in order to assist clinicians in clarifying the fertility status and thus providing the best treatment regime for patients.

\section{Conclusion}

Parents' stress during spermatogenesis and oogenesis can be critical for the health of the offspring. One of the probable reasons for these findings is the sensitivity of the gametes, in particular, male gametes, to environmental factors. The findings suggest that alterations caused by environmental factors in the gametes can affect the health of the children as epigenetics effects. We tried to show that the stress of spermatogenesis can affect the chromatin maturation of the sperm nucleus. It reduces the quality of the sperm and promotes the transfer of defective genes to the offspring. Therefore, to improve reproductive outcomes, stress-lowering interventions are better to be started before conception.

\section{Acknowledgments}

This study was supported by the Neurophysiology Research Center, Urmia University of Medical Sciences, Urmia, Iran.

\section{Conflict of Interest}

Authors declared no conflict of interest.

\section{References}

1. Moore IT, Jessop TS. Stress, reproduction, and adrenocortical modulation in amphibians and reptiles. Horm Behav 2003;43(1):39-47. [DOI:10.1016/S0018-506X(02)00038-7]

2. Curley J, Jensen C, Mashoodh R, Champagne F. Social influences on neurobiology and behavior: epigenetic effects during development. Psychoneuroendocrinology. 2011;36(3):352-71. [DOI:10.1016/j.psyneuen.2010.06.005] [PMID] [PMCID]

3. Heshmatian B, Roshan-Milani S, Saboory E. Prenatal Acute Stress Attenuated Epileptiform Activities in Neonate Mice. cell journal. 2010;12(1):81-6.

4. Saleh RA, Agarwal A, Nada EA, El-Tonsy MH, Sharma RK, Meyer A, et al. Negative effects of increased sperm DNA damage in relation to seminal oxidative stress in men with idiopathic and male factor infertility. Fertil Steril. 2003;79:1597-605. [DOI:10.1016/S0015-0282(03)00337-6]

5. Darnaudery M, Maccari S. Epigenetic programming of the stress response in male and female rats by prenatal restraint stress. Brain Res Rev. 2008;57(2):571-85. [DOI:10.1016/j.brainresrev.2007.11.004] [PMID]

6. Mahmoodkhani M, Saboory E, Roshan-Milani S, Azizi N, Karimipour M, Rasmi Y, et al. Pregestational stress attenuated fertility rate in dams and increased seizure susceptibility in offspring. 
Epilepsy Behav. 2018;79:174-9. [DOI:10.1016/i.yebeh.2017.12.016] [PMID]

7. Hashemi P, Roshan-Milani S, Saboory E, Ebrahimi L, Soltanineghad M. Interactive effects of prenatal exposure to restraint stress and alcohol on pentylenetetrazol-induced seizure behaviors in rat offspring. Alcohol. 2016;56:51-7. [DOI:10.1016/j.alcohol.2016.07.003] [PMID]

8. Stuppia L, Franzago M, Ballerini P, Gatta V, Antonucci I. Epigenetics and male reproduction: the consequences of paternal lifestyle on fertility, embryo development, and children lifetime health. Clin Epigenetics. 2015;7(1):120. [DOI:10.1186/s13148-015-0155-4] [PMID] [PMCID]

9. Larson-Cook KL, Brannian JD, Hansen KA, Kasperson KM, Aamold ET, Evenson DP. Relationship between the outcomes of assisted reproductive techniques and sperm DNA fragmentation as measured by the sperm chromatin structure assay. Fertil Steril 2003;80(4):895-902. [DOI:10.1016/S0015-0282(03)01116-6]

10. Mahmoodkhani M, Saboory E, Roshan-Milani S, Azizi N, Karimipour M, Sayyadi H, et al. Prepregnancy stress suppressed the reproductive systems in parents and changed sex ratio in offspring. J Appl Biomed. 2018;16(4):370-7. [DOI:10.1016/j.jab.2018.08.002]

11. Mangoli E, Talebi AR, Anvari M, Pourentezari M. Effects of experimentally-induced diabetes on sperm parameters and chromatin quality in mice. Iran $\mathbf{J}$ Reprod Med. 2013;11(1):53-60.

12. Sellami A, Chakroun N, Ben Zarrouk S, Sellami H, Kebaili S, Rebai T, et al. Assessment of chromatin maturity in human spermatozoa: useful aniline blue assay for routine diagnosis of male infertility. Adv Urol. 2013;2013. [DOI:10.1155/2013/578631] [PMID] [PMCID]

13. Kandratavicius L, Balista PA, Lopes-Aguiar C, Ruggiero RN, Umeoka EH, Garcia-Cairasco N, et al. Animal models of epilepsy: use and limitations. Neuropsychiatric disease and treatment. 2014;10:1693. [DOI:10.2147/NDT.S50371] [PMID] [PMCID]

14. Claycomb RJ, Hewett SJ, Hewett JA. Prophylactic, prandial rofecoxib treatment lacks efficacy against acute PTZ-induced seizure generation and kindling acquisition. Epilepsia. 2011;52(2):273-83. [DOI:10.1111/j.1528-1167.2010.02889.x] [PMID] [PMCID]

15. Gholami M, Saboory E, Zare S, Roshan-Milani S, Hajizadeh-Moghaddam A. The effect of dorsal hippocampal administration of nicotinic and muscarinic cholinergic ligands on pentylenetetrazolinduced generalized seizures in rats. Epilepsy Behav.
2012;25(2):244-9.

[DOI:10.1016/j.yebeh.2012.07.004] [PMID]

16. Gholipoor P, Saboory E, Roshan-Milani S, Fereidoni J. Effect of hyperthermia on histamine blood level and convulsive behavior in infant rats. Epilepsy Behav. 2013;29(2):269-74. [DOI:10.1016/j.yebeh.2013.07.026] [PMID]

17. Brinkworth MH. Paternal transmission of genetic damage: findings in animals and humans. Int $\mathrm{J}$ Androl 2000;23(3):123-35. [DOI:10.1046/j.13652605.2000.00222.x] [PMID]

18. Kundakovic M, Jaric I. The Epigenetic Link between Prenatal Adverse Environments and Neurodevelopmental Disorders. Genes. 2017;8(3):104. [DOI:10.3390/genes8030104] [PMID] [PMCID]

19. van Campen JS, Jansen FE, de Graan PN, Braun KP, Joels M. Early life stress in epilepsy: a seizure precipitant and risk factor for epileptogenesis. Epilepsy Behav. 2014;38:160-71. [DOI:10.1016/j.yebeh.2013.09.029] [PMID]

20. Dias BG, Ressler KJ. Parental olfactory experience influences behavior and neural structure in subsequent generations. Nat Neurosci 2014;17(1):89-96. [DOI:10.1038/nn.3594] [PMID] [PMCID]

21. Rodgers AB, Bale TL. Germ cell origins of posttraumatic stress disorder risk: the transgenerational impact of parental stress experience. Biol psychiatry. 2015;78(5):307-14 [DOI:10.1016/j.biopsych.2015.03.018] [PMID] [PMCID]

22. Gholipoor P, Saboory E, Ghazavi A, Kiyani A, Roshan-Milani S, Mohammadi S, et al. Prenatal stress potentiates febrile seizure and leads to longlasting increase in cortisol blood levels in children under 2 years old. Epilepsy Behav. 2017;72:22-7. [DOI:10.1016/i.yebeh.2017.04.021] [PMID]

23. Huang Y, Chen S, Xu H, Yu X, Lai H, Ho G, et al. Pre-gestational stress alters stress-response of pubertal offspring rat in sexually dimorphic and hemispherically asymmetric manner. BMC neuroscience. 2013;14(1):67. [DOI:10.1186/14712202-14-67] [PMID] [PMCID]

24. Huang Y, Shi X, Xu H, Yang H, Chen T, Chen S, et al. Chronic unpredictable stress before pregnancy reduce the expression of brain-derived neurotrophic factor and N-methyl-D-aspartate receptor in hippocampus of offspring rats associated with impairment of memory. Neurochem Res. 2010;35(7):1038-49. [DOI:10.1007/s11064-0100152-0] [PMID]

25. Aitken RJ, Baker MA, Sawyer D. Oxidative stress in the male germ line and its role in the aetiology of male infertility and genetic disease. Reprod Biomed 
Online. 2003;7(1):65-70. [DOI:10.1016/S14726483(10)61730-0]

26. Sirard M-A, Richard F, Blondin P, Robert C. Contribution of the oocyte to embryo quality. Theriogenology. 2006;65(1):126-36. [DOI:10.1016/j.theriogenology.2005.09.020] [PMID]

27. Frolova AI, Moley KH. Glucose transporters in the uterus: an analysis of tissue distribution and proposed physiological roles. Reproduction (Cambridge, England). 2011;142(2):211-20. [DOI:10.1530/REP11-0114] [PMID] [PMCID]

28. Ramakrishnan U, Grant F, Goldenberg T, Zongrone A, Martorell R. Effect of women's nutrition before and during early pregnancy on maternal and infant outcomes: a systematic review. Paediatr Perinat Epidemiol. 2012;26:285-301. [DOI:10.1111/j.13653016.2012.01281.x] [PMID]

29. Zimmermann MB. The effects of iodine deficiency in pregnancy and infancy. Paediatric and perinatal epidemiology. 2012;26:108-17. [DOI:10.1111/j.1365-3016.2012.01275.x] [PMID]

30. Lewis SE, Aitken RJ, Conner SJ, De Iuliis G, Evenson DP, Henkel R, et al. The impact of sperm DNA damage in assisted conception and beyond: recent advances in diagnosis and treatment. Reprod Biomed Online. 2013;27(4):325-37. [DOI:10.1016/j.rbmo.2013.06.014] [PMID]

31. Radwan M, Jurewicz J, Merecz-Kot D, Sobala W, Radwan P, Bochenek M, et al. Sperm DNA damagethe effect of stress and everyday life factors. Int $\mathrm{J}$ Impot Res. 2016;28(4):148. [DOI:10.1038/ijir.2016.15] [PMID]

32. Sabeti P, Pourmasumi S, Rahiminia T, Akyash F, Talebi AR. Etiologies of sperm oxidative stress. Int J Reprod Biomed (Yazd) 2016;14(4):231-40. [DOI:10.29252/ijrm.14.4.231]

33. Agarwal A, Virk G, Ong C, du Plessis SS. Effect of oxidative stress on male reproduction. World J Mens Health. 2014;32(1):1-17. [DOI:10.5534/wjmh.2014.32.1.1] [PMID] [PMCID]

34. Nasr-Esfahani MH, Razavi S, Tavalaee M. Failed fertilization after ICSI and spermiogenic defects. Fertility and sterility. 2008;89(4):892-8. [DOI:10.1016/j.fertnstert.2007.04.012] [PMID]

\section{How to Cite This Article:}

Saboory E, Mahmoodkani M, Roshan-Milani S, Rasmi Y. Effect of Predatory Stress Before Fertilization on Body Weight and Pentylenetetrazole-Induced Seizure in Rat Offspring. J Adv Med Biomed Res. 2020; 28 (127) :97-104

\section{Download citation:}

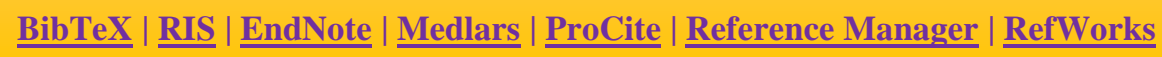

\section{Send citation to:}

Mendeley 2 Zotero $\underline{\underline{\text { DefWorks }}} \underline{\underline{\text { RefWorks }}}$ 